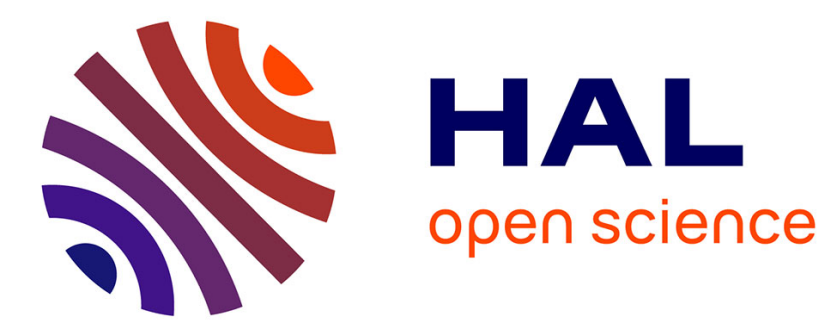

\title{
Etude d'un moteur à reluctance à feuilletage axial
}

M. Pakdel, G. Quichaud, J. Robert

\section{To cite this version:}

M. Pakdel, G. Quichaud, J. Robert. Etude d'un moteur à reluctance à feuilletage axial. Revue de Physique Appliquée, 1980, 15 (2), pp.331-339. 10.1051/rphysap:01980001502033100 . jpa-00244733

\section{HAL Id: jpa-00244733 https://hal.science/jpa-00244733}

Submitted on 1 Jan 1980

HAL is a multi-disciplinary open access archive for the deposit and dissemination of scientific research documents, whether they are published or not. The documents may come from teaching and research institutions in France or abroad, or from public or private research centers.
L'archive ouverte pluridisciplinaire HAL, est destinée au dépôt et à la diffusion de documents scientifiques de niveau recherche, publiés ou non, émanant des établissements d'enseignement et de recherche français ou étrangers, des laboratoires publics ou privés. 


\title{
Etude d'un moteur à reluctance à feuilletage axial
}

\author{
M. Pakdel, G. Quichaud et J. Robert \\ Laboratoire de Génie Electrique des Universités Paris VI et Paris XI $\left(^{*}\right)$ \\ E.S.E. Plateau du Moulon, 91190 Gif sur Yvette, France \\ (Reçu le 19 juin 1979, révisé le 22 octobre 1979, accepté le 25 octobre 1979)
}

\begin{abstract}
Résumé. - Les auteurs étudient de façon théorique et expérimentale les performances d'un moteur à reluctance muni d'un rotor feuilleté constitué de tôles planes parallèles et dirigées selon son axe. Ils développent une méthode prenant en compte les flux de fuites entre tôles et qui conduit à l'expression du couple de la machine. Cette expression est vérifiée expérimentalement et les performances des moteurs munis de divers types de rotors comparées. Les auteurs procèdent ensuite à l'étude du fonctionnement de ce moteur en régimes respectivement non autopiloté et autopiloté et établissent les diagrammes tension-courant correspondants.
\end{abstract}

\begin{abstract}
The authors investigate both theoretically and experimentally the performances of a reluctance engine. This laminated rotor is constituted of plane steel sheets parallel to the axis. The authors work out a method taking into consideration the leakage flux between sheets which leads to the expression of the motor torque. This expression is checked experimentally and the performances of motors using various rotor patterns are compared. In the second part of their paper, the authors study respectively the non self controlled and self controlled workings of this engine and draw the corresponding voltage-current diagrams.
\end{abstract}

1. Introduction. - Une machine à reluctance variable alimentée à fréquence fixe à partir du réseau présente des caractéristiques synchrones. Il en résulte pour l'utilisateur divers inconvénients tel que difficultés de démarrage, problèmes de stabilité de fonctionnement, nécessité de faibles inerties de charge.

En intercalant un système relevant de l'électronique industrielle entre le réseau et la machine, on confère à cette dernière un regain d'intérêt considérable. Les inconvénients dont il vient d'être question disparaissent avec l'auto-pilotage [1]. Dès lors, les machines à reluctance sont utilisables comme moteur d'entraînement à vitesse variable. Notons en outre que certaines configurations de machines à entrefer axial ou machines à disques imbriqués [2], conduisent à des puissances massiques très élevées particulièrement intéressantes pour des entraînements à basse vitesse.

Ces applications potentielles nouvelles des machines à reluctance rendent légitime la recherche d'un dimensionnement optimal et de performances accrues. Dans cet ordre d'idées, un feuilletage axial du rotor est équivalent à l'introduction d'une anisotropie de la perméabilité magnétique de ce dernier. Aussi nous proposons-nous dans le présent travail d'étudier

(*) Associé au C.N.R.S., № 127

REVUE DE PHYSIQUe APPLLQUÉE - T 15, № 2, FÉVRIER 1980 l'influence d'un tel feuilletage sur les performances de machines à reluctance à entrefer radial et de comparer ces performances à celles d'une machine à reluctance classique.

2. Etude élémentaire de l'influence d'un feuilletage axial du rotor sur le couple statique d'une machine à reluctance. - 2.1 POSITION DU PROBLÈME. STRUCTURES CONSIDÉRÉES ET CALCUL DU COUPLE STATIQUE DANS UN CAS IDÉAL. - Nous nous proposons de comparer les performances de machines cylindriques à reluctance, bipolaires, à champ tournant dont les rotors sont respectivement

- massif (structure classique, Fig. 1a)

- feuilleté axialement (Fig. 1b).

Dans un second temps, nous superposerons un feuilletage radial au feuilletage axial (feuilletage mixte).

Nous négligerons les effets d'extrémités et travaillerons dans les deux dimensions d'un plan sécant normal à l'axe de la machine.

Le stator rapporté à un système d'axes $O x y$ supporte un système de courants tournants dont la densité angulaire mesurée sur l'axe $O z$ de la machine a pour expression :

$$
j=-I_{0} \cos (u-\omega t), \quad I_{0}=\text { Cte }
$$




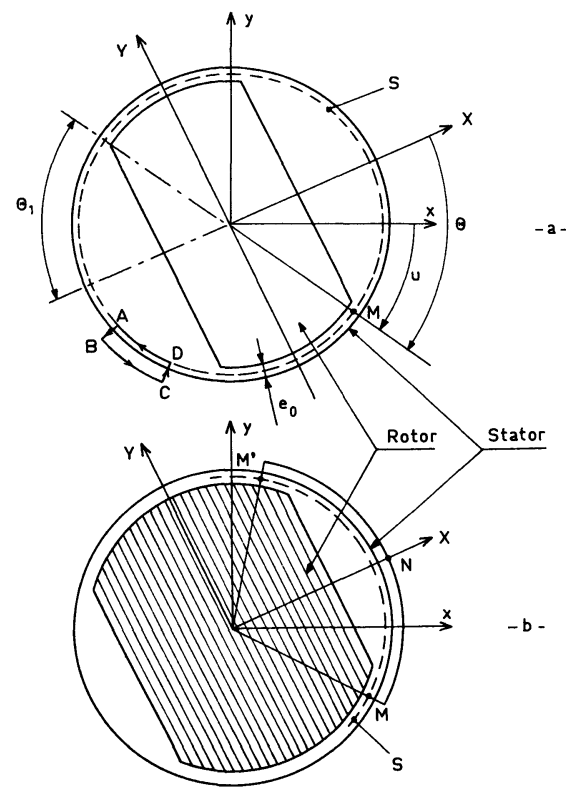

Fig. 1. - Définition des structures et des notations. $a$ ) rotor à reluctance classique; $b$ ) rotor à reluctance feuilleté axialement. [Definition of structures and notations. a) Classical rotor, b) axially laminated rotor.]

$u$ désigne l'abscisse angulaire d'un point courant $M$ de la surface interne du stator et $\omega$ la vitesse angulaire constante de la machine.

Le rotor est rapporté à un référentiel $O X Y$ dont la position par rapport au stator est définie par :

$$
\mathbf{O x}, \mathbf{O X}=\omega t-\theta_{0} .
$$

Dans ce nouveau repère, la densité de courant $j$ est indépendante du temps et s'exprime par

$$
j=-I_{0} \cos \left(\theta-\theta_{0}\right)
$$

où $\theta$ désigne la position angulaire du point $M$.

Nous adopterons les notations suivantes :

- $R, l_{1}$ : rayon et longueur du rotor,

- $e_{0}$ : largeur de l'entrefer dans sa partie constante,

- $2 \theta_{1}$ : extension angulaire de chaque méplat limitant le rotor.

La structure générale de la machine étant ainsi précisée, nous nous proposons d'évaluer les efforts exercés sur le rotor au moyen du tenseur des contraintes de Maxwell. Introduisons pour ce faire une surface cylindrique fictive $S$ liée au rotor et infiniment proche du stator (Fig. 1a). On sait que l'effort cherché s'exprime en fonction de l'excitation magnétique locale $\mathbf{H}$ et de l'élément de surface ds orienté vers l'intérieur de $S$ par la relation [3], [4] :

$$
\mathbf{d F}=\mu_{0}\left[\frac{H^{2}}{2} \mathbf{d s}-(\mathbf{H . d s}) \mathbf{H}\right]
$$

où $\mu_{0}$ est la perméabilité magnétique du vide.
On en déduit les expressions des contraintes normales et tangentielles s'exerçant sur $S$ :

$$
\begin{aligned}
& \left(\frac{\mathrm{d} F}{\mathrm{~d} s}\right)_{\mathrm{t}}=-\mu_{0} H_{\mathrm{t}} H_{\mathrm{n}} \\
& \left(\frac{\mathrm{d} F}{\mathrm{~d} s}\right)_{\mathrm{n}}=\mu_{0}\left(H_{\mathrm{t}}^{2}-H_{\mathrm{n}}^{2}\right)
\end{aligned}
$$

où $H_{\mathrm{t}}$ et $H_{\mathrm{n}}$ sont les composantes normales et tangentielles de $\mathbf{H}$ (relativement à $S$ ). Par intégration de la relation (5) sur $S$, on obtient l'expression du couple engendré sur l'arbre du rotor :

$$
\Gamma=\int_{0}^{2 \pi}-\mu_{0} H_{\mathrm{t}} H_{\mathrm{n}} R^{2} l_{1} \mathrm{~d} \theta .
$$

On détermine la composante tangentielle $H_{t}$, en appliquant le théorème d'Ampère le long du contour élémentaire $\mathrm{ABCD}$ de la figure $1 a$ :

$$
H_{\mathrm{t}}=\frac{I_{0}}{R} \cos \left(\theta-\theta_{0}\right) .
$$

On notera que cette expression est indépendante de la géométrie et de la structure du rotor.

2.2 Cas de la machine a feuilletage aXial. Le rotor est constitué par un empilage de tôles magnétiques minces de perméabilité suffisamment élevée pour pouvoir, au moins dans un premier temps, négliger le flux de fuite entre deux tôles. Nous néglirons également la composante normale de l'excitation dans les zones de l'entrefer où la largeur de ce dernier est variable.

Dans ces conditions, on aboutit en appliquant la loi de conservation du flux à une tôle et le théorème d'Ampère au circuit $\mathbf{M M}^{\prime} \mathrm{N}$ (Fig. $1 b$ ), aux relations suivantes :

$$
\begin{aligned}
H_{\mathrm{n}}(\theta)+H_{\mathrm{n}}(-\theta) & =0 \\
e_{0}\left[H_{\mathrm{n}}(\theta)-H_{\mathrm{n}}(-\theta)\right] & =2 I_{0} \cos \theta_{0} \sin \theta
\end{aligned}
$$

d'où nous tirons l'expression de la composante normale de l'excitation dans les zones où la largeur de l'entrefer est constante :

$$
H_{\mathrm{n}}(\theta)=\frac{I_{0}}{e_{0}} \cos \theta_{0} \sin \theta .
$$

En portant (8) et (11) dans (7), on obtient l'expression du couple

$$
\Gamma=\mu_{0} C\left(\theta_{1}\right) \frac{R l_{1} I_{0}^{2}}{2 \theta_{0}} I_{0}^{2} \sin 2 \theta_{0}
$$

avec

$$
\dot{C}\left(\theta_{1}\right)=\pi-2 \theta_{1}+\sin 2 \theta_{1} .
$$


2. 3 CAS DE la maChine a RELUCTANCE ClassiQue. COMPARAISON AVEC LA PRÉCÉDENTE. - Considérons la machine classique (Fig. 1a) excitée par le courant $j$. Les relations (9) et (10) deviennent :

$$
\begin{aligned}
\iint_{S} H_{\mathrm{n}} \mathrm{d} s & =0 \\
e_{0} \frac{\mathrm{d} H_{\mathrm{n}}}{\mathrm{d} \theta} & =-j
\end{aligned}
$$

et conduisent à

$$
H_{\mathrm{n}}=\frac{I_{0}}{e_{0}} \sin \left(\theta-\theta_{0}\right) .
$$

Enfin le couple s'écrit :

$$
\Gamma^{\prime}=\mu_{0} C^{\prime}\left(\theta_{1}\right) \frac{R l_{1} I_{0}^{2}}{2 e_{0}} \sin 2 \theta_{0}
$$

avec

$$
C^{\prime}\left(\theta_{1}\right)=2 \sin 2 \theta_{1} .
$$

Ainsi les couples $\Gamma$ et $\Gamma^{\prime}$ ne diffèrent que par les coefficients $C\left(\theta_{1}\right)$ et $C^{\prime}\left(\theta_{1}\right)$ dont nous avons porté les variations en fonction de $\theta_{1}$ sur la figure 2. Dans les conditions magnétiques idéales que nous avons définies, nous observons un gain de couple maximum voisin de $60 \%$ quand $\theta_{1}$ est nul. Cette valeur de $\theta_{1}$ qui correspond à une géométrie cylindrique ne peut être atteinte que dans le cas d'un rotor feuilleté.

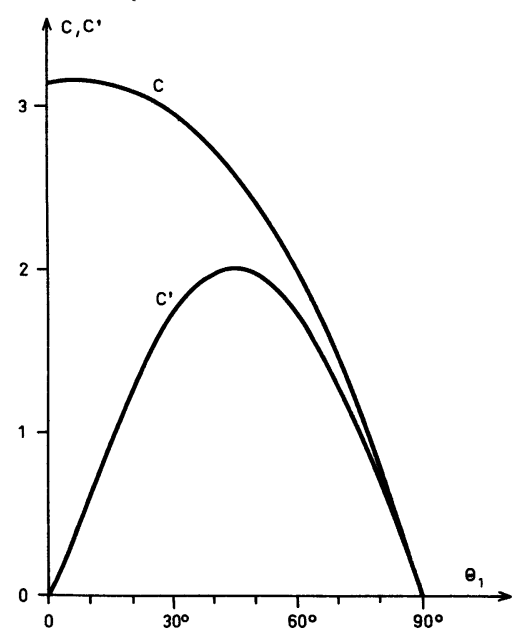

Fig. 2. - Variations des coefficients $C$ et $C^{\prime}$ en fonction de $\theta_{1}$. [Variations of the coefficients $C$ and $C^{\prime}$ versus $\theta_{1}$.]

3. Prise en compte du flux de fuites entre tôles. Le fait de négliger le flux de fuites entre tôles constitue une approximation qui n'est pas toujours légitime [5], [6]. Aussi avons-nous affiné les précédents calculs en tenant compte de ce flux de fuite [8].
3.1 GÉOMÉTRIE DU ROTOR. - La figure 3 est une vue en perspective du rotor étudié ; la figure 4 précise les notations. Soient :

- $2 N$, le nombre total de tôles numérotées de 1 à $2 N$.

- $l$, l'épaisseur uniforme de ces tôles.

- $l^{\prime}$, l'épaisseur uniforme du matériau isolant non magnétique séparant deux tôles à l'exception du couple $N ; N+1$.

- $\theta_{k}$ et $\theta_{k}^{\prime}$ les abscisses angulaires des arêtes de la $k$-ième tôle.

- $\varkappa_{1}$, l'angle définissant le profil d'une tôle.

- $2 h_{1}(y)$, l'épaisseur à l'abscisse $y$ de l'isolant séparant les tôles $N$ et $N+1$.

- $2 h_{1}$, la valeur maximum du paramètre précédent.

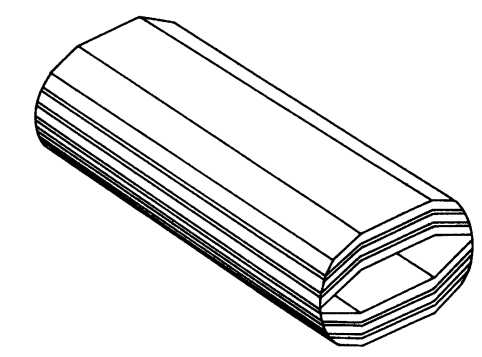

Fig. 3. - Vue de l'ensemble des tôles d'un rotor feuilleté axialement.

[General view of the steel sheets of an axially laminated rotor.]

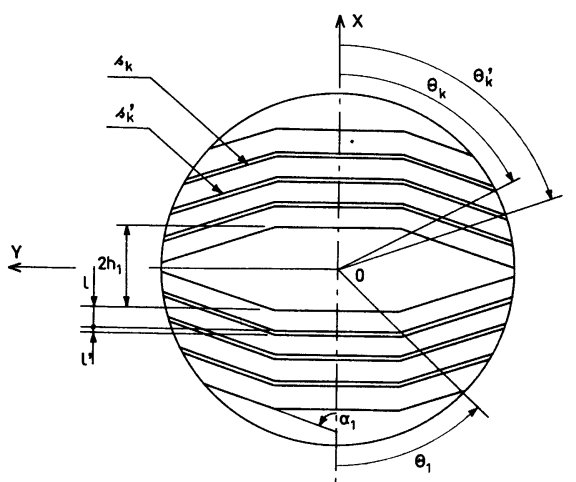

Fig. 4. - Paramètres géométriques définissant un rotor feuilleté axialement.

[Geometrical parameters defining an axially laminated rotor.]

3.2 PRINCIPE DE LA MISE EN ÉQUATION ET CALCUL DU COUPLE. - Une section droite du rotor admet son centre $O$ comme centre de symétrie. Ce point est également centre de symétrie pour les conditions aux limites sur $S$ puisque, d'après (8) :

$$
\mathbf{H}_{t}(\theta+\pi)=\mathbf{H}_{t}(\theta) \text {. }
$$

Si donc $P$ et $P^{\prime}$ sont deux points symétriques par rapport à $\mathrm{O}$, il vient

$$
\mathbf{H}(P)=\mathbf{H}\left(P^{\prime}\right)
$$


d'où, pour le potentiel magnétique $M$ :

$$
M(P)=-M\left(P^{\prime}\right)
$$

si nous supposons que $M$ est nul en $O$. Dans ces conditions, le potentiel magnétique est défini sur la surface d'analyse $S$ par les relations :

$$
\begin{aligned}
M(\theta) & =\int H_{\mathrm{t}}(\theta) R \mathrm{~d} \theta \\
M(\theta+\pi) & =-M(\theta)
\end{aligned}
$$

d'où nous tirons, compte tenu de (8) :

$$
M(\theta)=I_{0} \sin \left(\theta-\theta_{0}\right) .
$$

Comme précédemment, nous nous proposons de déterminer le couple électromagnétique d'une machine à reluctance munie du rotor étudié. Pour ce faire, nous considérons les potentiels magnétiques $M_{k}$ des $N$ premières tôles comme des inconnues auxiliaires et écrivons la conservation du flux au niveau de chaque tôle. Soient $s_{k}$ et $s_{k}^{\prime}$ les surfaces métalliques en contact avec l'isolant (Fig. 4), nous avons pour

$$
\begin{gathered}
2 \leqslant k \leqslant N-1: \\
\left(\frac{M_{k-1}-M_{k}}{l^{\prime}}\right) s_{k}-\left(\frac{M_{k}-M_{k+1}}{l^{\prime}}\right) s_{k}^{\prime}= \\
=\int_{\theta_{k}}^{\theta_{k}} \frac{M_{k}-M(\theta)}{e_{0}} R l_{1} \mathrm{~d} \theta+\int_{-\theta_{k}}^{\theta_{k}} \frac{M_{k}-M(\theta)}{e_{0}} R l_{1} \mathrm{~d} \theta .
\end{gathered}
$$

Pour la $1^{\text {re }}$ tôle, il faut écrire le nombre de gauche de cette équation

$$
\int_{-\theta_{1}^{\prime}}^{\theta_{1}} \frac{M(\theta)-M_{1}}{e(\theta)} R l_{1} \mathrm{~d} \theta-\left(\frac{M_{1}-M_{2}}{l^{\prime}}\right) s_{1}^{\prime} .
$$

On a de même pour la $n$-ième tôle

$$
\left(\frac{M_{n-1}-M_{n}}{l^{\prime}}\right) s_{N}-\int_{-R}^{R} \frac{M_{n}}{h_{1}(y)} l_{1} \mathrm{~d} y .
$$

Le système des $N$ équations peut être condensé sous la forme matricielle

$$
[A][M]=[E]
$$

où $[M]$ est la matrice colonne des $N$ inconnues $M_{k}$ et $[A]$ une matrice carrée tridiagonale de la forme :

$$
\left[\begin{array}{cccccccc}
A_{11} & A_{12} & 0 & 0 & 0 & \vdots & 0 & 0 \\
A_{21} & A_{22} & A_{23} & 0 & 0 & \vdots & 0 & 0 \\
0 & A_{32} & A_{33} & A_{34} & 0 & \vdots & 0 & 0 \\
0 & 0 & A_{43} & A_{44} & A_{45} \vdots & 0 & 0 \\
\ldots \ldots & \ldots & \ldots & \ldots & \ldots \ldots \ldots \ldots \ldots & \ldots \\
0 & 0 & 0 & 0 & 0 & A_{N N-1} & A_{N N}
\end{array}\right]
$$

dont l'inversion est simplifiée même quand $N$ est grand.
Les potentiels magnétiques $M_{k}$ étant calculés à partir de (24), la relation

$$
H_{k}(\theta)=\frac{M_{k}-M(\theta)}{e_{0}}
$$

permet d'obtenir la composante radiale de l'excitation sur $S$. Notons que cette relation est valable pour $|\theta|<\theta_{1}^{\prime}$ (Fig. 4), en y faisant $k=1$ et en remplaçant $e_{0} \operatorname{par} e(\theta)$.

Seul le fondamental de $H_{n}$ est nécessaire pour calculer le couple à partir de (7). Nous avons calculé ce fondamental à partir des expressions précédentes et l'avons mis sous la forme :

$H_{n_{\mathrm{F}}}(\theta)=\frac{I_{0}}{\pi e_{0}}\left(C_{\mathrm{d}} \cos \theta_{0} \sin \theta-C_{\mathrm{q}} \sin \theta_{0} \cos \theta\right)$

où $C_{\mathrm{d}}$ et $C_{\mathrm{q}}$ sont calculés à partir des $M_{k}$. La relation (7) conduit alors à l'expression du couple moteur :

$$
\Gamma=\left(C_{\mathrm{d}}-C_{\mathrm{q}}\right) \frac{\mu_{0} R l_{1}}{2 e_{0}} I_{0}^{2} \sin 2 \theta_{0}
$$

3.3 RÉSUltats THÉORIQUes. - Nous avons comparé deux rotors feuilletés de caractéristiques géométriques communes

$$
R=31 \mathrm{~mm} \quad e_{0}=0,3 \mathrm{~mm} \quad \theta_{2}=\frac{\pi}{2}
$$

le premier étant constitué de tôles minces $(l=1 \mathrm{~mm}$, $\left.l^{\prime}=0,1 \mathrm{~mm}\right)$, le second de tôles épaisses $(N=1$, $h_{1}=10 \mathrm{~mm}$ ). Il s'agit dans ce dernier cas d'un rotor dit à plots [7], dont une coupe est donnée sur la figure 5 . En négligeant le flux de fuite entre les 2 plots ainsi que sur les méplats, on trouve que :

$C_{\mathrm{d}}-C_{\mathrm{q}}=\frac{4}{\theta_{2}-\theta_{1}}\left(\sin \theta_{2}-\sin \theta_{1}\right)+$

$$
+2\left(\sin 2 \theta_{1}-\sin 2 \theta_{2}\right) \text {. }
$$

Les résultats des calculs effectués sur ordinateur sont consignés sur la figure 6 , dont l'examen conduit aux remarques suivantes :

- Seule une machine à feuilletage axial mince permet d'obtenir le couple maximum correspondant

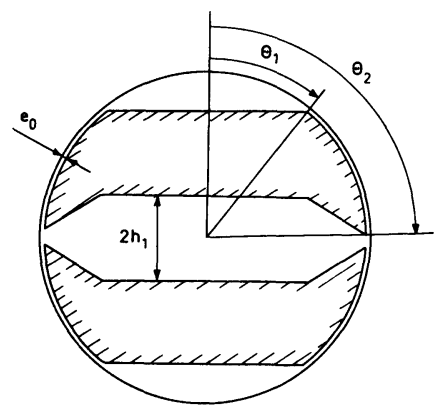

Fig. 5. - Géométrie d'un rotor à plots.

[Segmental rotor.] 


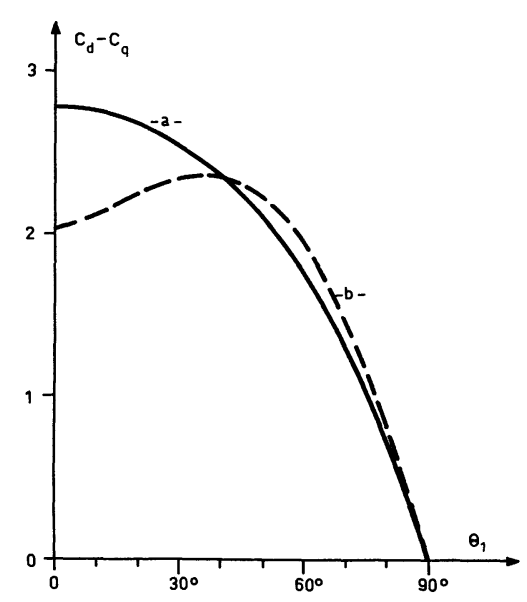

Fig. 6. - Variations du coefficient du couple en fonction de l'extension angulaire $\theta_{1}$ des méplats. $a$ ) Rotor à feuilletage axial; b) rotor à plots.

[Variation of torque coefficient versus flat parts angular extension $\left.\theta_{1} . a\right)$ Axially laminated rotor; $b$ ) segmental rotor.]

à l'utilisation de toute la surface du rotor $\left(\theta_{1}=0\right)$, tandis que pour un rotor à plots le couple maximum est obtenu pour $\theta_{1}=35^{\circ}$.

- Pour $\theta_{1}<35^{\circ}$, les couples respectifs des machines à feuilletage mince et à plots sont comparables et toujours supérieurs à celui d'une machine classique. Le gain maximum réel est de $40 \%$, alors que nous l'avions évalué à $60 \%$ en négligeant les fuites entre tôles.

La figure 7 traduit l'influence de l'épaisseur relative des tôles et de l'isolant sur le couple. L'optimum est obtenu pour un coefficient de remplissage

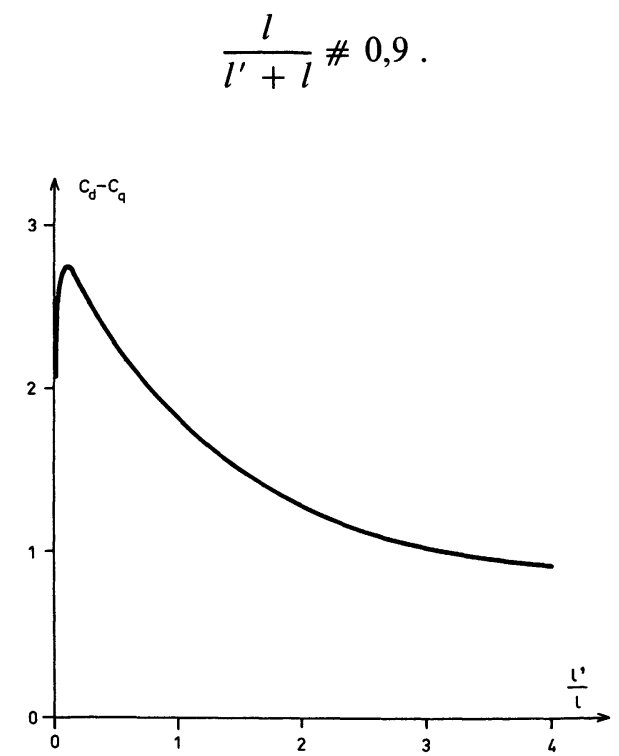

Fig. 7. - Variation du coefficient de couple en fonction du rapport des épaisseurs respectives des isolants et des tôles.

[Variation of torque coefficient versus the ratio of the respective thickness of insulators and steel sheets.]
4. Caractéristiques théoriques des machines à feuilletage axial. - 4.1 DÉTERMINATION DE L'IMPÉDANCE SYNCHRONE. - En utilisant la relation (25) nous avons été conduits à déterminer l'expression suivante de l'impédance synchrone d'une machine à feuilletage axial :

$$
\bar{Z}=r+\frac{j}{2}\left(X_{\mathrm{d}}-X_{\mathrm{q}}\right) \exp \left(-2 j \theta_{0}\right)+\frac{j}{2}\left(X_{\mathrm{d}}+X_{\mathrm{q}}\right)
$$

où $r$ est la résistance d'une phase. Les réactances synchrones longitudinale $X_{\mathrm{d}}$ et transversale $X_{\mathrm{q}}$ sont telles que :

$$
\begin{aligned}
& X_{\mathrm{d}}=L_{\mathrm{d}} \omega=1+L_{0} C_{\mathrm{d}} \omega \\
& X_{\mathrm{q}}=L_{\mathrm{q}} \omega=1+L_{0} C_{\mathrm{q}} \omega
\end{aligned}
$$

où $l$ est l'inductance de fuite d'une phase et $L_{0}$ a pour expression :

$$
L_{0}=\frac{\mu_{0} n_{0}^{2} l_{1} R}{l_{0}}
$$

$n_{0}$ désigne la densité linéique maximale de conducteurs de chaque bobinage de l'enroulement statorique supposé diphasé. Enfin $\theta_{0}$ représente le retard angulaire de l'axe longitudinal du rotor (parallèle au feuilletage) sur l'axe de la f.m.m. tournante due aux courants statoriques (Fig. 8). Ce dernier coïncide avec l'axe du bobinage équivalent tournant.

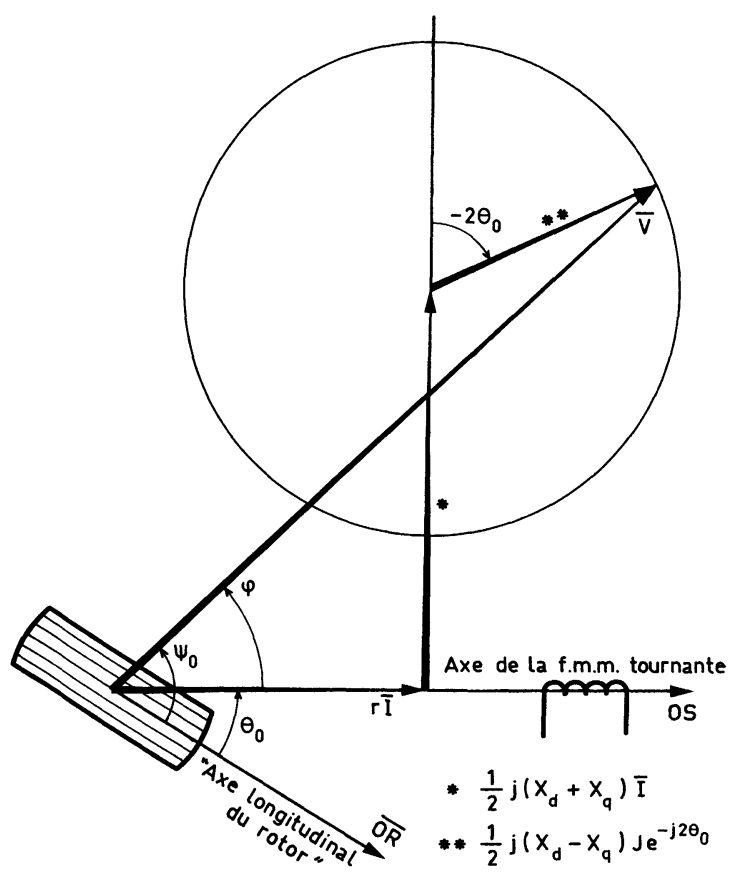

Fig. 8. - Diagramme de la tension d'un moteur à reluctance non autopiloté alimenté à courant constant et fréquence fixe.

[Voltage diagram of a non self controlled reluctance engine fed with constant current at invariable frequency.] 
Aussi si $\bar{V}$ et $\bar{I}$ sont les amplitudes complexes des tensions et courants d'une phase de la machine, nous avons d'après (27) :

$$
\bar{V}=\bar{Z} \cdot \bar{I} .
$$

Il apparaît que $\bar{V}$ décrit un cercle lorsque $\theta_{0}$ varie si $\bar{I}$ est constant (Fig. 8). Notons également que dans ces conditions l'expression du couple développé par la machine se met sous la forme :

$$
\Gamma=\frac{X_{\mathrm{d}}-X_{\mathrm{q}}}{\omega} I^{2} \sin 2 \theta_{0}=\left(L_{\mathrm{d}}-L_{\mathrm{q}}\right) I^{2} \sin \theta_{0} .
$$

4.2 Diagramme CIRCUlaire DU COURANT D'UNE MACHINE ALIMENTÉE EN TENSION. - Dans le cas d'une machine alimentée sous tension constante, l'angle $\psi_{0}$ de cette dernière par rapport à l'axe longitudinal du rotor constitue le paramètre de réglage. On a (Fig. 8) :

$$
\operatorname{tg} \psi_{0}=\frac{\operatorname{tg} \theta_{0}+\operatorname{tg} \varphi_{\mathrm{d}}}{1-\operatorname{tg} \theta_{0} \operatorname{tg} \varphi_{\mathrm{q}}}
$$

où

$$
\operatorname{tg} \varphi_{\mathrm{d}}=\frac{X_{\mathrm{d}}}{r} \quad \operatorname{tg} \varphi_{\mathrm{q}}=\frac{X_{\mathrm{q}}}{r}
$$

Il est commode d'inverser, compte tenu de

$$
\bar{I}=\left[g-\frac{j}{2}\left(y_{\mathrm{d}}-y_{\mathrm{q}}\right) \exp \left(-2 j \psi_{0}\right)-\frac{j}{2}\left(y_{\mathrm{d}}+y_{\mathrm{q}}\right)\right] \bar{V}
$$

avec

$$
\begin{aligned}
g & =\frac{r}{r^{2}+X_{\mathrm{d}} X_{\mathrm{q}}}, \quad y_{\mathrm{d}}=\frac{X_{\mathrm{d}}}{r^{2}+X_{\mathrm{d}} X_{\mathrm{q}}}, \\
y_{\mathrm{q}} & =\frac{X_{\mathrm{q}}}{r^{2}+X_{\mathrm{d}} X_{\mathrm{q}}} .
\end{aligned}
$$

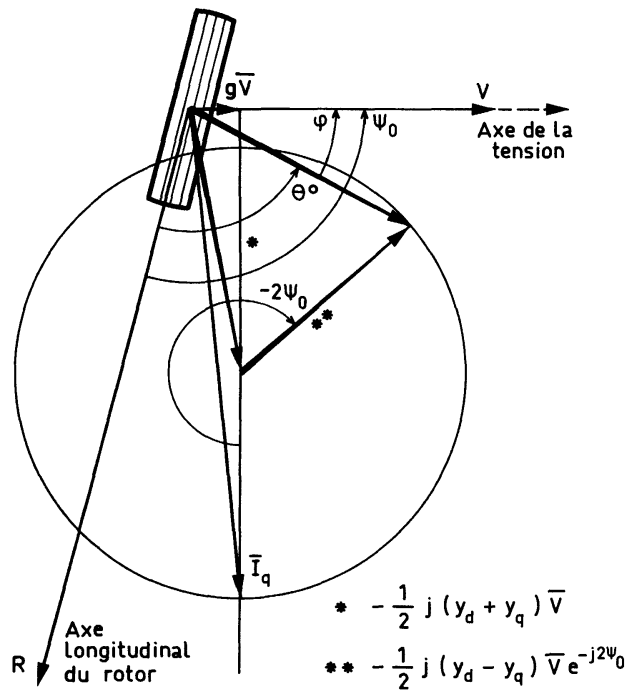

Fig. 9. - Diagramme du courant d'un moteur à reluctance non autopiloté, alimenté à tension constante et fréquence fixe.

[Current diagram of a non self controlled reluctance engine fed with constant voltage at invariable frequency.]
Ce système de relations conduit au diagramme circulaire du courant de la figure 9 .

Le couple s'écrit :

$$
\begin{gathered}
\Gamma=K \frac{V^{2}}{\omega}\left(\frac{1}{X_{\mathrm{q}}}-\frac{1}{X_{\mathrm{d}}}\right) \sin \left(\psi_{\mathrm{o}}-\varphi_{\mathrm{d}}\right) \cos \left(\psi_{0}-\varphi_{\mathrm{q}}\right) \\
K=2 \frac{\left[\left(1+\operatorname{tg}^{2} \varphi_{\mathrm{d}}\right)\left(1+\operatorname{tg}^{2} \varphi_{\mathrm{q}}\right)\right]^{1 / 2}}{\operatorname{tg} \varphi_{\mathrm{d}} \operatorname{tg} \varphi_{\mathrm{q}}+\left(\operatorname{tg} \varphi_{\mathrm{d}} \operatorname{tg} \varphi_{\mathrm{q}}\right)^{-1}}
\end{gathered}
$$

Les variations de $\Gamma$ en fonction de $\psi_{0}$ à diverses fréquences sont précisées sur la figure 10 , les données étant les suivantes :

$$
\begin{aligned}
V & =51 \mathrm{~V}, \quad L_{\mathrm{d}} & =0,39 \mathrm{H}, \\
L_{\mathrm{q}} & =0,1 \mathrm{H}, \quad r & =6,7 \Omega .
\end{aligned}
$$

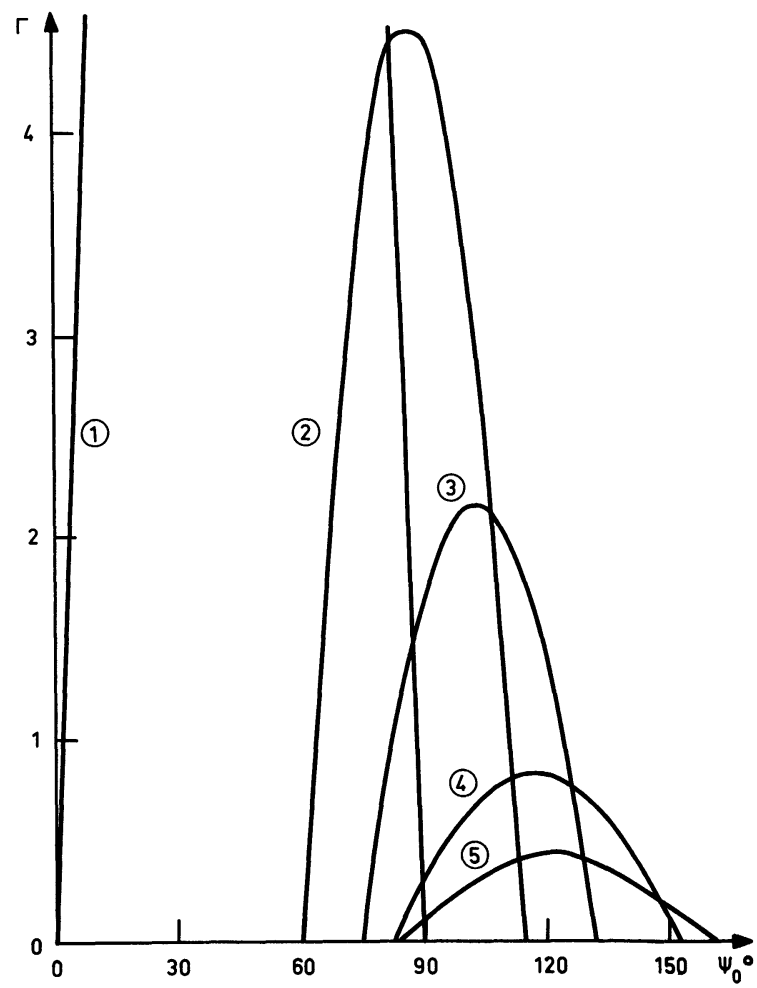

Fig. 10. - Variations en fonction de $\psi_{0}$ du couple d'une machine à feuilletage axial alimentée sous tension constante pour diverses fréquences. 1. $f=0 \mathrm{~Hz} ; 2 . f=5 \mathrm{~Hz} ; 3 . f=10 \mathrm{~Hz} ; 4$. $f=20 \mathrm{~Hz} ; 5 . f=30 \mathrm{~Hz}$.

[Torque variations of an axially laminated engine versus $\psi_{0}$ at constant voltage and various frequencies. $1 . f=0 \mathrm{~Hz} ; 2$. $f=5 \mathrm{~Hz} ; 3 . f=10 \mathrm{~Hz} ; 4 . f=20 \mathrm{~Hz} ; 5 . f=30 \mathrm{~Hz}$.]

4.3 MACHINE AUTOPILOTÉE ALIMENTÉE EN TENSION. - Au paragraphe 5.1 suivant nous décrivons une réalisation d'une machine autopilotée alimentée en tension. Comme nous le verrons, pour une telle machine $\psi_{0}$ est imposé par le calage du rotor par rapport à la tension $\bar{V}$ d'alimentation nécessairement variable. Le diagramme circulaire du courant (Fig. 8) 
n'est plus valable. Nous avons montré que l'extrémité du courant $\bar{I}$ décrit une ellipse [6]. Les composantes directe $I_{\mathrm{d}}$ et en quadrature $I_{\mathrm{q}}$ de $I$ sont respectivement données par

$$
\begin{aligned}
& I_{\mathrm{d}}=I \cos \theta_{0}=\frac{V}{r} \frac{\cos \psi_{0}+K_{\mathrm{q}} \omega \sin \psi_{0}}{1+K_{\mathrm{d}} K_{\mathrm{q}} \omega^{2}} \\
& I_{\mathrm{q}}=I \sin \theta_{0}=\frac{V}{r} \frac{\sin \psi_{0}-K_{\mathrm{d}} \omega \cos \psi_{0}}{1+K_{\mathrm{d}} K_{\mathrm{q}} \omega^{2}}
\end{aligned}
$$

avec

$$
K_{\mathrm{d}}=\frac{L_{\mathrm{d}}}{r} \quad K_{\mathrm{q}}=\frac{L_{\mathrm{q}}}{r} .
$$

De tels diagrammes elliptiques sont tracés sur la figure 11 pour diverses valeurs de $\psi_{0}$. On notera que le fonctionnement en moteur correspond à $\theta_{0}$ positif.

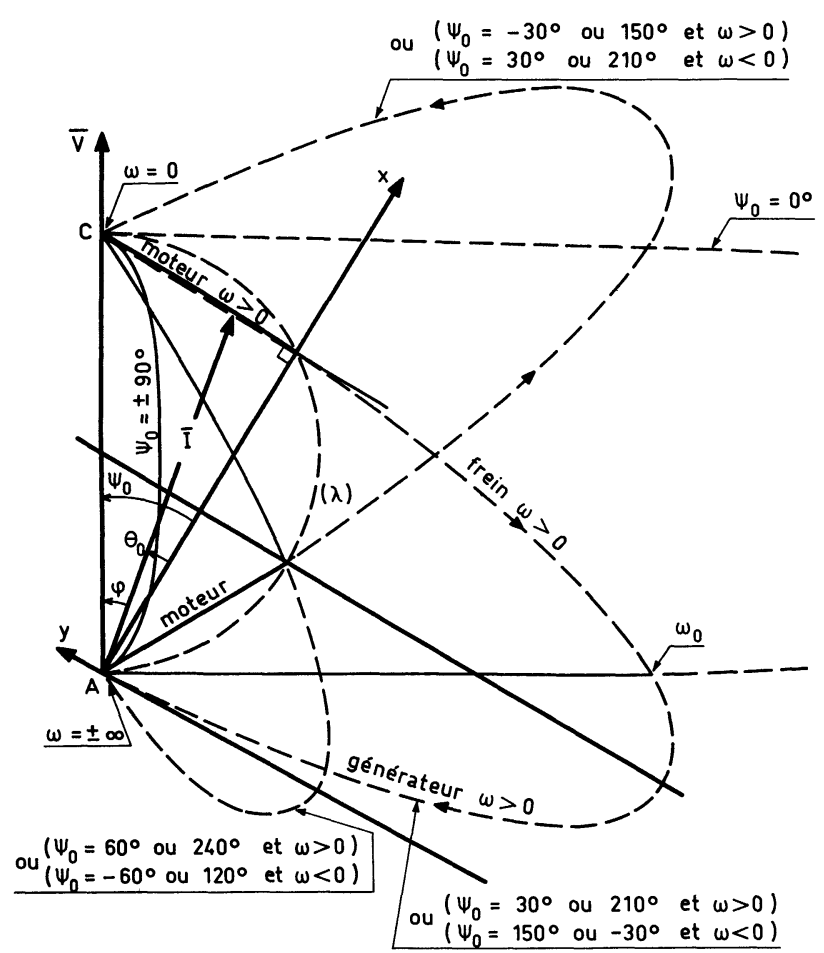

Fig. 11. - Diagramme du courant d'une machine autopilotée alimentée sous tension constante.

[Current diagram of a self controlled engine fed with constant voltage.]

L'expression du couple en fonction de la pulsation de rotation est obtenue à partir de (35)

$$
\begin{aligned}
\Gamma=\frac{2 V^{2} \cos ^{2} \psi_{0}}{r}\left(K_{\mathrm{d}}-K_{\mathrm{q}}\right) \times \\
\quad \times \frac{\left(\operatorname{tg} \psi_{0}-K_{\mathrm{d}} \omega\right)\left(1+K_{\mathrm{q}} \omega \operatorname{tg} \psi_{0}\right)}{1+K_{\mathrm{d}} K_{\mathrm{q}} \omega^{2}} .
\end{aligned}
$$

Nous avons tracé sur la figure 12 le réseau de caractéristiques mécaniques en conservant, pour les para-

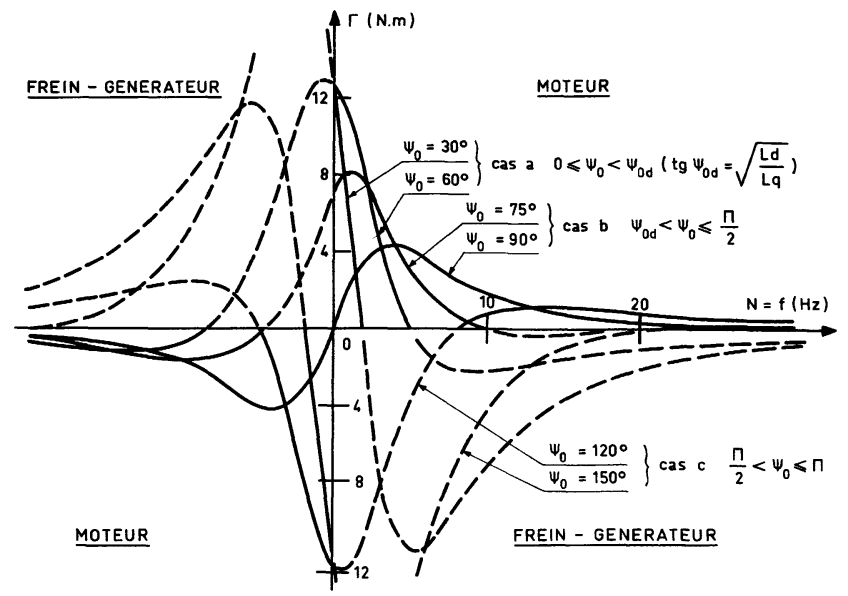

Fig. 12. - Variation du couple avec la vitesse d'une machine autopilotée alimentée sous tension constante.

[Torque variations versus speed of a self controlled engine fed with constant voltage.]

mètres du moteur et la tension d'alimentation, les valeurs définies lors du tracé de la figure 10 .

Nous constatons que :

- le moteur ne peut démarrer seul que pour $\psi_{0}<\frac{\pi}{2}$,

- le couple de démarrage est maximum pour

$$
\psi_{0}<\operatorname{Arctg} \sqrt{\frac{L_{\mathbf{d}}}{L_{\mathbf{q}}}}
$$

- la vitesse maximale est théoriquement infinie pour $\psi_{0}>\frac{\pi}{2}$.

Nous avons tracé en pointillé les caractéristiques relatives à un fonctionnement en générateur obtenu à faible vitesse pour $\psi_{0}>\frac{\pi}{2}$ et à grande vitesse pour $\psi_{0}<\frac{\pi}{2}$.

5. Réalisations pratiques et études expérimentales. 5.1 PrinCiPe ET RÉAlisation D'UN MOTEUR A RELUCTANCE AUTOPILOTÉ. - Afin de vérifier la validité de

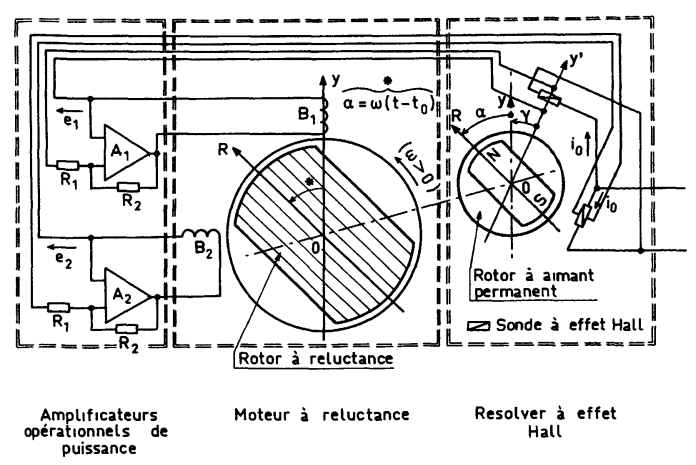

Fig. 13. - Schéma du montage d'un moteur autopiloté.

[Diagram of self controlled engine wiring.] 
ces résultats théoriques, nous avons réalisé un moteur à reluctance autopiloté dont le principe est précisé sur la figure 13. Dans un stator de machine asynchrone diphasée nous avons placé divers rotors à reluctance à feuilletage axial ou classique [6]. Un résolver à effet Hall (trigonomètre sinus-cosinus) dont le rotor à aimant permanent est lié rigidement au rotor à reluctance fournit les deux tensions de Hall :

$$
\begin{aligned}
& e_{1}=k \cos (\alpha-\gamma) \\
& e_{2}=k \sin (\alpha-\gamma) .
\end{aligned}
$$

La constante $k$ est proportionnelle au courant de commande des sondes à effet Hall. Lors d'une rotation uniforme :

$$
\alpha=\omega\left(t-t_{0}\right)
$$

nous obtenons deux tensions en quadrature qui alimentent par l'intermédiaire de deux amplificateurs opérationnels de puissance les phases du stator. Le calage $\gamma$ des sondes de Hall définit le retard $\psi_{0}$ de l'axe longitudinal du rotor par rapport à la tension. Dans ce qui suit, nous décrivons les résultats relatifs aux fonctionnements de cette machine munie de chacun des trois rotors de la figure 14. L'un est classique (avec $2 \theta_{1}=90^{\circ}$ ). Le second est à feuilletage simplement axial; le dernier est à feuilletage mixte axial radial. Le feuilletage radial est, pour des raisons constructives, assez grossier. Il est destiné à diminuer les pertes Joule rotor dues aux harmoniques du champ magnétique. En effet les tôles utilisées sont en acier extra-doux dont la résistivité est plus faible que celle des tôles au silicium. Les caractéristiques communes des 3 rotors sont les suivantes:

$$
\begin{aligned}
& \text { diamètre } 2 R_{2}=62,2 \mathrm{~mm}, \\
& \text { longueur utile } l_{1}=85 \mathrm{~mm} .
\end{aligned}
$$

Enfin l'épaisseur de l'entrefer est de 0,3 mm.

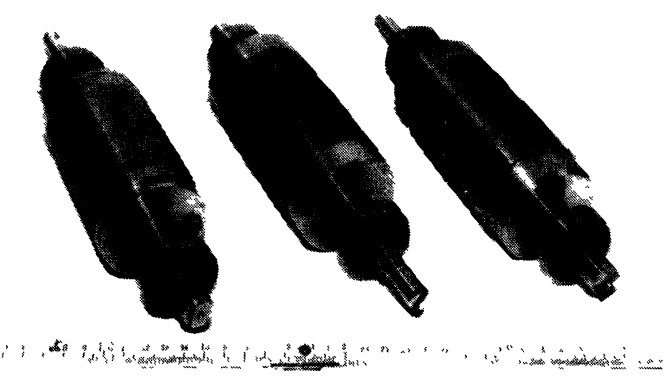

Fig. 14. - Rotors expérimentés. De gauche à droite, classique. à feuilletage axial, à feuilletage mixte.

[Tested rotors. From left to right, classical, axially laminated, mixed laminated.]

5.2 RéSUltats EXPÉRIMENTAUX. - Nous avons porté sur la figure 15 les couples statiques mesurés, la machine étant munie de chacun des 3 rotors précé-

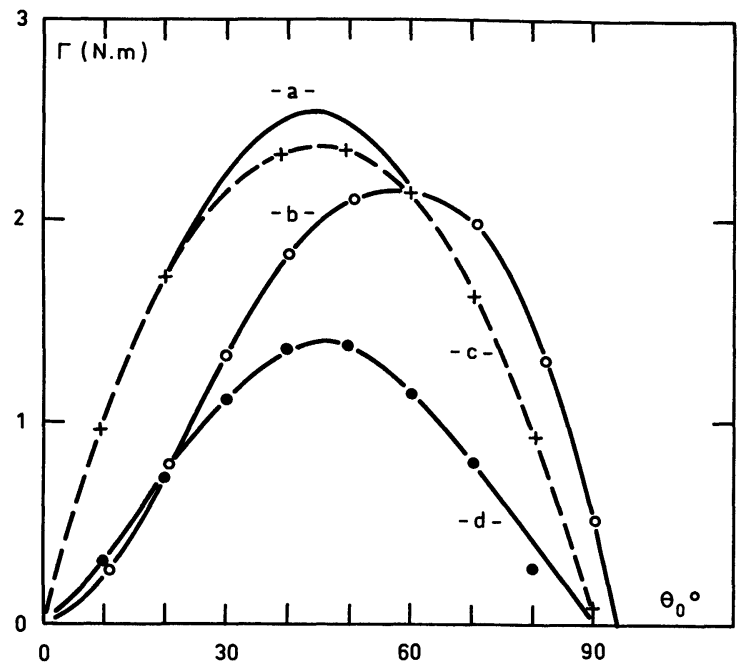

Fig. 15. - Couple statique d'une machine autopilotée alimentée en tension. a) Calculé pour un rotor à feuilletage axial ; $b$ ) mesuré pour un rotor à feuilletage axial; $c$ ) mesuré pour un rotor à feuilletage mixte; $d$ ) mesuré pour un rotor classique.

[Static torque of a self controlled engine fed with voltage. $a$ ) Computed for an axially laminated rotor; $b$ ) measured for an axially laminated rotor; $c$ ) measured for a mixed laminated rotor; $d$ ) measured for a classical rotor.]

dents et alimentée dans les mêmes conditions, ainsi que le couple statique calculé dans le cas du rotor à feuilletage axial par rapport aux machines à reluccouple du rotor à feuilletage axial. La déformation de la courbe de couple du rotor à feuilletage axial est probablement due à une dissymétrie géométrique. L'ensemble de ces mesures confirme les résultats théoriques en démontrant la supériorité des machines munies d'un rotor à feuilletage axial par rapport aux machines à réluctance classiques.

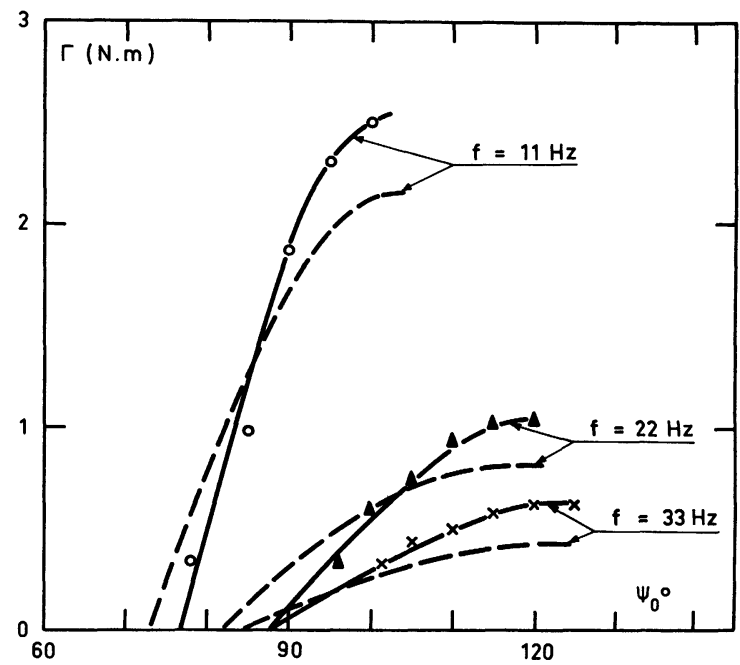

Fig. 16. - Caractéristiques de couples $\Gamma\left(\psi_{0}\right)$ du moteur non autopiloté pour diverses valeurs de la fréquence d'alimentation. $\longrightarrow$ courbe expérimentale; --- courbe théorique.

[Torque curves $\Gamma\left(\psi_{0}\right)$ of non self controlled engine for various values of frequency. tical curve.] 


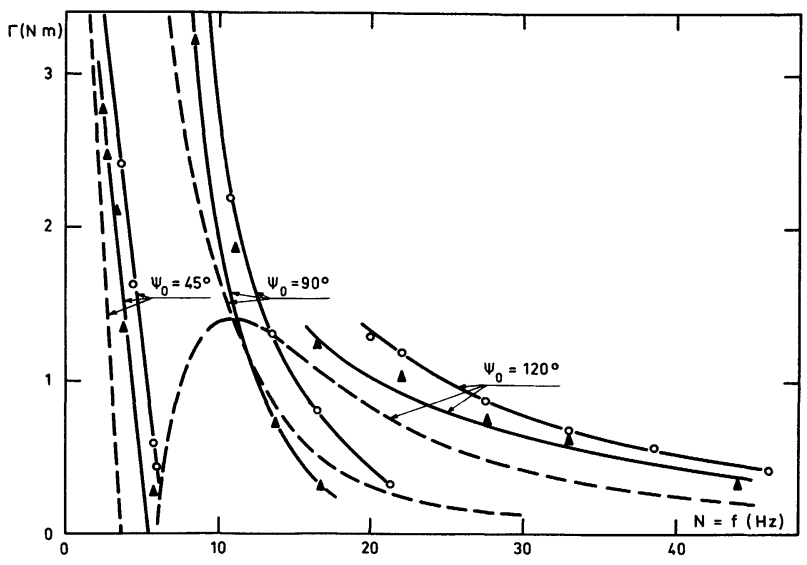

Fig. 17. - Caractéristiques couple-vitesse du moteur autopiloté. - $\mathrm{O}$ - mesurées pour le moteur à feuilletage mixte; $-\boldsymbol{\Delta}-$ mesurées pour le moteur à feuilletage axial; --- théorique.

[Speed-torque curves of self controlled engine. $-\mathrm{O}-$ measured for mixed lamination engine; $-\Delta-$ measured for axially laminated engine ; $-\longrightarrow$ theoretical.]

Sur les figures 16 et 17 nous avons comparé les couples dynamiques mesurés et calculés, les machines alimentées sous $51 \mathrm{~V}$ fonctionnant en moteur. La première figure est relative au moteur à feuilletage axial non autopiloté alimenté à fréquence variable, la seconde au moteur autopiloté muni de chacun des deux rotors feuilletés. Nous avons reporté sur ces figures les résultats théoriques précédemment établis (Figs. 10 et 12).

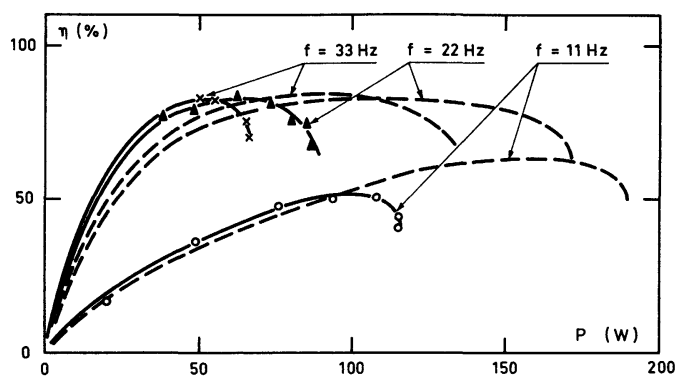

Fig. 18. - Variations du rendement d'un moteur non autopiloté en fonction de la puissance absorbée. —_ à rotor classique; - - à feuilletage mixte.

[Variations of non self controlled engine efficiency versus absorbed power. classical rotor ; - - mixed lamination.]
Enfin, nous avons porté sur les figures 18 et 19 le rendement et le facteur de puissance mesurés en fonction de la puissance absorbée d'un moteur non autopiloté.

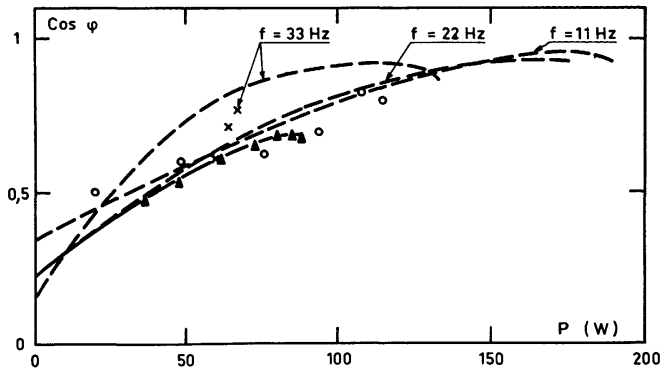

Fig. 19. - Variations du facteur de puissance d'un moteur non autopiloté en fonction de la puissance absorbée. Pour un moteur classique $\circ f=11 \mathrm{~Hz} ; \Delta f=22 \mathrm{~Hz} ; \mathrm{X} f=33 \mathrm{~Hz} ;-\longrightarrow$ moteur à feuilletage mixte.

[Variations of non self controlled power factor versus absorbed power. Classical rotor $O f=11 \mathrm{~Hz} ; \Delta f=22 \mathrm{~Hz} ; \mathrm{X} f=33 \mathrm{~Hz}$; - - mixed lamination.]

L'accord entre les mesures et les considérations théoriques que nous avons développées est satisfaisant, si l'on tient compte des imprécisions qui affectent la détermination de certains paramètres de la machine, l'entrefer en particulier. C'est probablement pour cette raison que sur la figure 17, les couples mesurés sont supérieurs aux couples calculés.

6. Conclusion. - La supériorité prévue théoriquement des machines à reluctance munies d'un rotor feuilleté axialement est confirmée par l'expérience.

Un tel résultat devrait encourager les physiciens à étudier d'autres types de machines à reluctance, dans lesquelles seraient introduites des anisotropies de perméabilité magnétique. Tel pourrait être le cas des machines à disques imbriqués déjà mentionnées pour lesquelles une optimisation des pertes magnétiques conduira sans doute à feuilleter les plots au moyen de fils magnétiques. Notre étude donne à penser que l'anisotropie ainsi introduite ne provoquera pas de diminution du couple électromagnétique.

\section{Bibliographie}

[1] Faucher, Lajoie Mazenc, Azizi-Ghannadi, Proc. of the International Conference on Electrical Machines Bruxelles $11,12,13$ septembre 1978

[2] Goyet, R., Jablon, C., Rioux, C., Compte rendu final du rapport DGRST no 75.7.1236, octobre 1976.

[3] Rioux, C., Théorie générale comparative des machines électriques, établie à partir des expressions du champ électromagnétique. RGE 79 (1970) 415.

[4] Rıoux, C., Thèse de docteur ès-science, Paris, 1964.

[5] Cruickshank, A. J. O., Anderson, A. F., Menzies, R. N. : Theory and performance of reluctance motors with axially laminated anisotropic rotors. Proc. IEE 118 (1971) 887.

[6] PAKDEL, M., Thèse de docteur ingénieur. Université Paris VI, 1978.

[7] Lawrenson, P. J., Agu, L. A., Theory and performance of polyphase reluctance machine. Proc. IEEE 111 (1964) 1435.

[8] Pakdel, M., Quichaud, G., Robert, J., Influence of an axial lamination on the performance of a reluctance machine. Proc. of the "International conference on electrical machines » Bruxelles 11, 12, 13 septembre 1978. 